\title{
CENTERS OF CONVEX SUBSETS OF BUILDINGS
}

\author{
ANDREAS BALSER AND ALEXANDER LYTCHAK
}

ABSTRACT. We prove that two dimensional convex subsets of spherical buildings are either buildings or have a center.

\section{INTRODUCTION}

Recently, convex cores of isometric group actions on symmetric spaces were studied in $[\mathrm{KL}$. The results of Kleiner and Leeb imply the following: Assume that the group $\Gamma$ acts by isometries on a symmetric space $H$ in a non-elementary way (i.e. without fixed point at infinity). Then the convex core of $\Gamma$ is a direct product of symmetric and Gromov-hyperbolic spaces. In particular the boundary at infinity of the convex core must be a spherical building with respect to the Tits metric.

This gives rise to the following more abstract question. Let $\Gamma$ be a group acting by isometries on a spherical building $G$. Let $X$ be a $\pi$-convex subset of $G$ invariant under $\Gamma$. Is it true that $\Gamma$ must have a fixed point if $X$ is not a building? In the case of small dimensions (of the building or at least the subset) we answer this question in the affirmative.

Theorem 1.1. Let $G$ be a spherical building, $X \subset G$ a convex subset. If $\operatorname{dim}(X) \leq 2$ then either $X$ is itself a building or the isometry group of $X$ has a fixed point in $X$.

In the proof we use some observations about general $C A T(1)$ spaces, which we consider to be of independent interest. In important special situations they were obtained in FNS04 by different methods.

The next result is shown in [LS97, Thm. B] for $r<\frac{\pi}{2}$ and in [FNS04, Thm. 1.7] for $\operatorname{diam} X=\frac{\pi}{2}$.

Proposition 1.2. Let $X$ be a finite-dimensional $C A T(1)$ space. If the radius $r$ of $X$ is smaller than $\pi$, then for the diameter of $X$ holds $\operatorname{diam}(X)>\operatorname{rad}(X)$.

1991 Mathematics Subject Classification. 53C20.

Key words and phrases. Isometry groups, fixed points, Alexandrov spaces, CAT(1) spaces, buildings. 
From this, an easy limiting argument gives the following uniform result, which follows from [LS97, Thm. B] in the case $r<\frac{\pi}{2}$ :

Theorem 1.3. For each natural number $n$ and each $r<\pi$ there is some $\varepsilon=\varepsilon(r, n)>0$, such that for each $C A T(1)$ space $X$ of dimension at most $n$, of radius at most $r$ with at least two points, we have $\frac{\operatorname{diam}(X)}{\operatorname{rad}(X)} \geq$ $1+\varepsilon$.

Remark 1.1. For $\frac{\pi}{2} \leq r<\pi$, this result can not be achieved by the methods of [LS97]. It is not clear to us what the optimal value of $\varepsilon$ should be and what the spaces with smallest possible ratio $\frac{\operatorname{diam}(X)}{\operatorname{rad}(X)} \operatorname{look}$ like.

In [LS97, Thm. B] it is shown that a CAT(1) space with radius $<\frac{\pi}{2}$ has a unique circumcenter, that is therefore fixed by the whole isometry group. In the compact case the next result is proved in [FNS04, Prop. 5.7].

Proposition 1.4. Let $X$ be a CAT(1) space of finite dimension and of radius $r \leq \frac{\pi}{2}$. Then $X$ has a circumcenter which is fixed by every isometry of $X$.

Remark 1.2. In both propositions the assumption of finite dimensionality is essential, compare [FNS04, pg. 5] or Example 3.1.

We explain the idea of the proof of Theorem 1.1 in the case where $X$ is compact. In this case we consider a smallest closed convex subset $Y$ of $X$ invariant under the isometry group of $X$ and one can assume that $Y$ is not a building. Since $Y$ is minimal one can not cut off any small neighborhood of any point $y \in Y$, such that the remaining subset is still convex (see Section 4 for more on the notion of nonremovable points). In dimension 2 this implies that each point is an inner point of some geodesic. Now we consider two points $y, z \in Y$ with maximal distance in $Y$. If $d(y, z)=\pi$ then $Y$ contains a circle and one can deduce that $\operatorname{rad}(Y)=\frac{\pi}{2}$. If $d(y, z)<\pi$, we show that one can choose geodesics through $y$ resp. through $z$. Using these, we construct a spherical quadrangle in which one of the diagonals is longer than $d(y, z)$, a contradiction. The last argument in the proof uses spherical quadrangles (not triangles as usual) and breaks down if the dimension is bigger than 2 .

We thank Bernhard Leeb for posing the question.

\section{Preliminaries}

2.1. Notations. By $d$ we denote distances in metric spaces. By $B_{r}(x)$ we will denote the closed metric ball of radius $r$ around the point $x$. For 
a point $x$ in a metric space $X$ we set $\operatorname{rad}_{x}(X):=\sup _{z \in X} d(x, z)$. By the radius resp. the diameter of $X$ we denote $\operatorname{rad}(X):=\inf _{x \in X} \operatorname{rad}_{x}(X)$ resp. $\operatorname{diam}(X):=\sup _{x \in X} \operatorname{rad}_{x}(X)$.

Since we are dealing with non-proper spaces we will use the concept of ultraconvergence (instead of the Gromov-Hausdorff convergence) with respect to a fixed non-principal ultrafilter $\omega$. We refer to [BH99, pp. 77-80] for details, see also [Lyt04, sect. 11]. We will denote by $\lim _{\omega}\left(X_{i}, x_{i}\right)$ the ultralimit of pointed metric spaces $X_{i}$ with respect to $\omega$. By $X^{\omega}$ we will denote the ultraproduct of $X$, i.e. the ultralimit of the constant sequence $(X, x)$.

2.2. CAT(1) spaces. A complete metric space is called CAT(1) if each pair of points with distance $<\pi$ is connected by a geodesic and all triangles of perimeter less than $2 \pi$ are not thicker than in $S^{2}$. We refer to BH99, ch. II] for the theory of such spaces. In a CAT(1) space $X$ we will denote by $S_{x}$ the link at the point $x$. A subset $C$ of a CAT(1) space is convex if all points in $C$ with distance $<\pi$ are joined by a geodesic in $C$.

By $\operatorname{dim}(X)$ we denote the geometric dimension of $X$ studied in Kle99. Ultralimits of CAT(1) spaces are CAT(1) and the dimension does not increase by this procedure ([Lyt04, L. 11.1]).

A subset $T$ of a CAT(1) space $X$ is spherical if it can be isometrically embedded into some Euclidean sphere. See BL04 for more on this.

2.3. Buildings and their convex subsets. We refer to KL97, sect. 3] for an account on spherical buildings. From the geometry of buildings we will only use the following basic property:

Let $G$ be a building. Then for each $x \in X$ there is a number $r_{x}>0$ (set $r_{x}$ so that any point $\bar{x} \in X$ with $d(\bar{x}, x)<r_{x}$ lies in a Weyl chamber containing $x$ ), such that for all $x, y \in X$ and all $\bar{x} \in B_{r_{x}}(x)$ and $\bar{y} \in B_{r_{y}}(y)$ the convex hull of the four points $x, \bar{x}, y, \bar{y}$ is a spherical (usually three-dimensional) subset of $G$. Remark that this property is inherited by convex subsets of buildings. We refer to BL04 for a more thorough study.

We will use the following result derived in BL04]:

Lemma 2.1 ([BL04, $6.3+6.4]$ ). Let $X$ be a convex subset of some building $G$. If $X$ is not a building, then $\operatorname{rad}(X)<\pi$. If $X$ contains an isometrically embedded $S^{n-1}$ and $\operatorname{dim}(X) \leq n$, then either $X$ is a building or $\operatorname{rad}(X)=\frac{\pi}{2}$. 


\section{RADil of CAT(1) SPACES}

3.1. Generalities. The following lemma follows from Lyt04, L. 11.7], but we give a direct proof here:

Lemma 3.1. Let $X$ be an $n$-dimensional $C A T(1)$ space, and let $S \subset X$ be an embedded $S^{n}$. Then for each $x \in X$ there is an antipode $y \in S$, i.e. a point satisfying $d(x, y) \geq \pi$. Therefore we have $\operatorname{rad}(X) \geq \pi$.

Proof. If $\operatorname{dim} S=0$, the claim is clear. Choose an arbitrary $y \in S$. Let $v \in S_{y}$ be the starting direction of $y x$. By induction, we can choose an antipode $w$ of $v$ in $S^{n-2}=S_{y} S \subset S_{y} X$. Now we obtain an antipode of $x$ by extending $x y$ inside $S$ in the direction of $w$.

The proof of the next lemma is a typical application of ultraproducts.

Lemma 3.2. Let $X$ be a $C A T(1)$ space. If $r=\operatorname{diam}(X)=\operatorname{rad}(X)<$ $\pi$, then for each point $x \in X^{\omega}$ holds $\operatorname{rad}\left(S_{x}\left(X^{\omega}\right)\right) \leq \frac{\pi}{2}$.

Proof. Let $x=\left(x_{i}\right)$. Choose $z_{i} \in X$ with $d\left(z_{i}, x_{i}\right) \geq r-\frac{1}{i}$. For $z=\left(z_{i}\right) \in X^{\omega}$ we get $d(z, x)=r=\operatorname{diam}(X)=\operatorname{diam}\left(X^{\omega}\right)$. From the CAT(1) property we immediately obtain that the starting direction $v \in S_{x}$ of the geodesic $x z$ satisfies $d(v, w) \leq \frac{\pi}{2}$ for each $w \in S_{x}$.

Proof of Proposition [1.2. In Kle99, Thm. B] it is shown that in each CAT(1) space $X$ of dimension $n+1$, some link $S_{x}$ contains an $n$ dimensional Euclidean sphere. Using Lemma 3.2 and Lemma 3.1, we deduce Proposition 1.2.

Proof of Theorem 1.3. Assume the contrary and choose a sequence of spaces $X_{i}$ with $\frac{\operatorname{diam}\left(X_{i}\right)}{\operatorname{rad}\left(X_{i}\right)} \rightarrow 1$. Since we assume that the spaces have more than one point we may rescale $X_{i}$ (without leaving the category of at most $n$-dimensional $C A T(1)$ spaces) and assume that the radius of all the spaces $X_{i}$ is equal to $r$. For the ultralimit space $X=\lim _{\omega}\left(X_{i}\right)$ we obtain $\operatorname{rad}(X)=\operatorname{diam}(X)=r$ in contradiction to Proposition [1.2. since the dimension of $X$ is bounded by $n$ too.

Example 3.1. The subset $P$ of points in the Hilbertsphere with all coordinates non-negative satisfies $\operatorname{rad}(P)=\operatorname{diam}(P)=\frac{\pi}{2}$. Moreover, since swapping coordinates is an isometry, the isometry group of $P$ has no fixed points, thus the assumption of finite-dimensionality is essential in Proposition 1.2 and Proposition 1.4. (This was also observed in [FNS04, pg. 5]). Moreover $P$ contains points $x$ with $\operatorname{rad}\left(S_{x} P\right)=\pi$ (let $x$ be a point with all components positive). 


\subsection{CAT(1) spaces of radius $\frac{\pi}{2}$. We recall}

Definition 3.1. A point $x$ in a metric space $X$ is a circumcenter of $X$ if $\operatorname{rad}_{x}(X)=\operatorname{rad} X$ holds.

Due to [LS97, Thm. B] a CAT(1) space $X$ of radius $<\frac{\pi}{2}$ has a unique circumcenter. As a first step, we extend the existence result:

Lemma 3.3. Let $X$ be a CAT(1) space of finite dimension and radius $\frac{\pi}{2}$. Then $X$ has a circumcenter.

Proof. By definition, we find a sequence of points $x_{i} \in X$ such that $\operatorname{rad}_{x_{i}}(X) \rightarrow \frac{\pi}{2}$. This sequence defines a circumcenter $x=\left(x_{i}\right)$ for the ultraproduct $X^{\omega}$, which is a space of the same dimension as $X$ (Lyt04, Cor. 11.2]). We have $d(x, X) \leq \frac{\pi}{2}$. In the case of equality we have $d(x, y)=\frac{\pi}{2}$ for all $y \in X$, and the convex hull of $x$ and $X$ in $X^{\omega}$ would be isometric to the spherical join $X *\{x\}$ ([Lyt04, L. 4.1]). This contradicts $\operatorname{dim}\left(X^{\omega}\right)=\operatorname{dim}(X)$.

Hence, there is a unique projection $x^{\prime}$ of $x$ to $X$ ([BH99, II.2.6.1]). Triangle comparison shows that $x^{\prime}$ is a circumcenter of $X$.

Proof of Proposition 1.4. The proof can be concluded in the same manner as in the proof of [FNS04, Thm. 1.3]. The set $X_{1}$ of all circumcenters of $X$ is (by the above) a non-empty subset of $X$, which is closed, convex and invariant under the isometry group. Moreover by definition the diameter of $X_{1}$ is not not bigger than $\operatorname{rad}(X)=\frac{\pi}{2}$.

Due to Proposition 1.2 the radius of $X_{1}$ is smaller than $\frac{\pi}{2}$ and due to [LS97, Thm. B], the space $X_{1}$ has a unique circumcenter, that is therefore fixed by the isometry group of $X$.

\section{Groups acting on a CAT(1) space}

4.1. Removable points. For $r>0$ we will denote by $S_{r}(x)$ the sphere of radius $r$ around $x$, i.e. the set of all points $y$ with $d(y, x)=r$.

Remark 4.1. Do not confuse the sphere $S_{r}(x)$ with the link $S_{x}$.

Definition 4.1. Let $X$ be a CAT(1) space and $r>0$. A point $x \in X$ is called $r$-removable, if the closed convex hull of the sphere $S_{r}(x)$ does not contain $x$. It is called removable if it is $r$-removable for all $r>0$. A point which is not removable is called non-removable.

Remark that if $x \in X$ is $r$-removable then it is $r^{\prime}$-removable for all $r^{\prime}>r$. A point $x \in X$ is removable iff it is removable inside some ball $B_{r}(x)$. The following examples should only illustrate the notion and will not be used in the proof below. 
Example 4.2. In every geodesically complete CAT(1) space each point is non-removable.

Example 4.3. A point $x \in X$ is non-removable if it is inner point of some geodesic or, more general, if it is an inner point of some geodesic in the ultraproduct $X^{\omega}$. If $X$ has dimension 1 the last condition is also necessary.

Example 4.4. For every CAT(1) space $Z$, each point in the spherical join $X=Z * S^{1}$ is a midpoint of a geodesic of length $\pi$, hence it is not $r$-removable for $r<\frac{\pi}{2}$.

Example 4.5. A closed convex subset $S$ of a finite-dimensional sphere has no removable points iff $S$ has the form $S=Z * S^{1}$.

Example 4.6. Let $X$ be a locally conical space ([BL04, sect. 3.2]) with $\operatorname{dim}(X) \leq 2$. A point $x \in X$ is non-removable iff $x$ is an inner point of some geodesic $\gamma \subset X^{\omega}$ in the ultraproduct $X^{\omega}$ of $X$.

Example 4.7. Let $X$ be a closed convex subset in a Riemannian manifold of constant curvature. A point $x \in X$ is not removable iff it is the inner point of some geodesic. An interesting question which we conjecture to be true is whether this statement is also true if $X$ is a Riemannian manifold with variable curvature.

Unfortunately it is difficult to say much about the behavior of convex hulls and therefore of removable points under ultralimits. The following easy observation will be used below

Lemma 4.1. Let $\left(X_{i}, o_{i}\right)$ be CAT(1) spaces, $(X, o)=\lim _{\omega}\left(X_{i}, o_{i}\right)$. Consider a point $x=\left(x_{i}\right) \in X$ such that for some fixed $s>0$ the point $x_{i}$ is not s-removable in $X_{i}$. Then for each $m \in X$ with $l=$ $d(m, x) \leq \frac{\pi}{2}$, there is a point $q \in X$ with $d(q, x)=s$ and $d(m, q) \geq$ $d(m, x)$.

Proof. We can represent $m$ as a sequence of points $m=\left(m_{i}\right)$, with $m_{i} \in X_{i}, l_{i}=d\left(m_{i}, x_{i}\right)<\frac{\pi}{2}$. We may assume that $m \neq x$ and hence, that $l_{i} \not \rightarrow 0$. Since $x_{i}$ is not $s$-removable, there must be at least one point $q_{i}$ with $d\left(q_{i}, x_{i}\right)=s$, that is not contained in the closed convex ball $B_{l_{i}-\rho_{i}}\left(m_{i}\right)$, for each $\rho_{i}>0$. If we choose $\rho_{i} \rightarrow 0$, then the point $q=\left(q_{i}\right) \in X$ has the desired properties.

4.2. Minimal invariant subsets. Non-removable points are related to minimal invariant subsets by the following observation:

Lemma 4.2. Let $X$ be a $C A T(1)$ space, $x \in X, \Gamma$ a group of isometries of $X$. Assume that there is no proper closed, convex, and $\Gamma$-invariant 
subset of $X$ containing $x$. Then each removable point of $X$ is contained in the closure of the orbit $\overline{\Gamma x}$. More precisely if $d(z, \Gamma x)=\varepsilon$, then $z$ is not $r$-removable for $r<\varepsilon$.

Proof. Assume the contrary and consider the closed convex convex hull $C$ of $X \backslash B_{r}(z)$. Then $C$ does not contain $z$ but $x \in g C$ for $g \in \Gamma$. Considering $\cap_{g \in \Gamma} g C$ we get a contradiction to the minimality of $X$.

Remark 4.8. If $X$ is a minimal closed convex subset invariant under $\Gamma$, then either $X$ has no removable points or $\Gamma$ has a dense orbit. If $X$ is finite dimensional and not discrete one can show that in the latter case each point is non-removable as well.

4.3. Centers. We will stick to the following

Definition 4.2. We say that a CAT(1) space $X$ has a center if its group of isometries $\Gamma^{X}=I \operatorname{so}(X)$ has a fixed point.

A group operating isometrically on a CAT(1) space $X$ has a fixed point iff it has an orbit of diameter $<\frac{\pi}{2}$. This implies

Lemma 4.3. Let $X_{i}$ be a sequence of CAT(1) spaces. If $X_{i}$ has no center, then $X=\lim _{\omega} X_{i}$ has no center too.

\section{The Proof of the Main Theorem}

For the proof of Theorem 1.1 the following lemma is crucial:

Lemma 5.1. Let $Y$ be a convex subset of a building, $\operatorname{dim}(Y) \leq 2$. Let $y, z \in Y$ be inner points of some geodesics in $Y$. If $d(y, z)=\operatorname{diam}(Y)$ holds, then $d(y, z)=\pi$ and $Y$ contains some $S^{1}$.

Proof. Let $y$ resp. $z$ be inner points of geodesics $p_{1} p_{2}$ resp. $q_{1} q_{2}$. We may assume that $p_{i} \in B_{r_{y}}(y)$ resp. $q_{i} \in B_{r_{z}}(z)$ for $i=1,2$, where $r_{y}$ resp. $r_{z}$ are the conicality radii at $y$ resp. at $z$ (see Subsection 2.3). If $d(y, z)=\pi$, the convex hull of $q_{1} q_{2}$ and $y$ is a circle $S^{1}$.

Thus assume that $d(y, z)<\pi$. For the geodesic $\eta=y z$ we deduce from the assumption $d(y, z)=\operatorname{diam}(Y)$, that $\eta$ meets $y p_{i}$ and $z q_{j}$ orthogonally. From the geometry of buildings we derive (cf. Subsection 2.3 again) that the convex hulls of $y p_{i}$ and $z q_{j}$ are spherical for $i, j=1,2$. Since $\operatorname{dim}(Y) \leq 2$ these spherical hulls are 2-dimensional spherical quadrangles.

Now in the two dimensional sphere the following statement holds: For $i=1,2$ let $\bar{\gamma}_{i}$ be geodesics in $S^{2}$ starting in $x_{i}$ with $d\left(x_{1}, x_{2}\right)<\pi$. Assume that $\bar{\eta}=x_{1} x_{2}$ meets $\bar{\gamma}_{1}$ and $\bar{\gamma}_{2}$ orthogonally. If $\bar{\gamma}_{1}$ and $\bar{\gamma}_{2}$ are on different sides of $\eta$, i.e. if $\angle_{x_{1}}\left(\bar{\gamma}_{1}(\varepsilon), \bar{\gamma}_{2}(\varepsilon)\right)>\frac{\pi}{2}$, then $d\left(\bar{\gamma}_{1}(\varepsilon), \bar{\gamma}_{2}(\varepsilon)\right)>$ $d\left(x_{1}, x_{2}\right)$ for small $\varepsilon>0$. 
Therefore we get a contradiction to $d(y, z)=\operatorname{diam}(Y)$ as soon as we can verify that $\angle_{y}\left(p_{i}, q_{j}\right)>\frac{\pi}{2}$ for some $i$ and $j$. However from the one-dimensionality of $S_{y} Y$ we directly obtain that $\angle_{y}\left(p_{1}, q_{j}\right)>\frac{\pi}{2}$ for $j=1$ or $j=2$.

Now we are going to prove Theorem 1.1 along the line explained in the introduction. The non-compactness causes additional difficulties.

Proof of Theorem 1.1. Let $G$ be a spherical building modelled on the Coxeter group $W$. Let $X \subset G$ be a convex subset of dimension at most 2 and without center. Assume finally that $X$ is not a building. Due to Lemma 2.1] we have $\bar{r}:=\operatorname{rad}(X)<\pi$.

Let $C_{W}^{\bar{r}}$ be the class of all CAT(1) spaces that have dimension at most 2 , no center, whose radius is at most $\bar{r}$ and that admit an isometric embedding into some spherical building modelled on the Coxeter group $W$. Since an ultralimit of buildings modelled on $W$ is again a building modelled on $W$, the class $C_{W}^{\bar{r}}$ is closed under ultralimits (by Lemma 4.3). Therefore we can find a space $Z$ in $C_{W}^{\bar{r}}$, whose radius $r \leq \bar{r}$ is the smallest possible for spaces in $C_{W}^{\bar{r}}$ (let $r_{i} \rightarrow r$ be radii of elements of $C_{W}^{\bar{r}}$, and let $Z$ be the ultralimit of the corresponding spaces).

Replacing $Z$ by its ultraproduct $Z^{\omega}$ we may assume that there is a point $x \in Z$ with $\operatorname{rad}_{x}(Z)=\operatorname{rad}(Z)$. Let $Y$ be the smallest closed convex subset of $Z$ containing $x$ and invariant under the isometry group $\Gamma^{Z}$ of $Z$. Clearly, a center for $Y$ would also be a center for $Z$, thus $Y$ is in $C_{W}^{r}$ too. Moreover we have $\operatorname{rad} Y=\operatorname{rad}_{x}(Y)=r$ by the minimality of $r$, and $r>\frac{\pi}{2}$ by Prop. 1.4 since $Y$ has no center.

From Proposition 1.2 we derive diam $Y-\operatorname{rad}_{x}(Y)=2 \varepsilon>0$. Therefore for points $y_{i}, z_{i} \in Y$ with $d\left(y_{i}, z_{i}\right) \rightarrow \operatorname{diam}(Y)$ we can apply Lemma 4.2 and see that for all big $i$ the points $y_{i}$ and $z_{i}$ are not $\varepsilon$-removable.

Consider $y=\left(y_{i}\right), z=\left(z_{i}\right) \in Y^{\omega}$. We have $d(y, z)=\operatorname{diam} Y^{\omega}=$ $\operatorname{diam} Y$. Assume that $y$ and $z$ are inner points of some geodesics in $Y^{\omega}$. Then from Lemma 5.1 we derive that $Y^{\omega}$ contains a circle, from Lemma 2.1 we see that $\operatorname{rad}\left(Y^{\omega}\right)=\frac{\pi}{2}$ and by Proposition 1.4 the space $Y^{\omega}$ must have a center - a contradiction.

By symmetry it is enough to prove that $y$ is an inner point of a geodesic. Decreasing $\varepsilon$ we may assume that it is smaller than the conicality radius $r_{y}$ of $y$ in $Y^{\omega}$.

Denote by $\eta$ the geodesic between $y$ and $z$ and let $v \in S_{y}$ be its starting direction. From $d(y, z)=\operatorname{diam} Y^{\omega}$ we derive $\operatorname{rad}_{v}\left(S_{y}\right) \leq \frac{\pi}{2}$.

Set $m=\eta\left(\frac{\pi}{2}\right)$. By Lemma 4.1 there is a point $q$ with $d(q, m) \geq \frac{\pi}{2}$ and $d(y, m)=\varepsilon$. Since the triangle $y m q$ is spherical, for the starting direction $w$ of $y q$ we obtain $d(v, w)=\frac{\pi}{2}$. 
Now we consider the point $z_{\delta}$ on the geodesic $q z$ with $d\left(z, z_{\delta}\right)=\delta \rightarrow$ 0 . As above we derive from Lemma 4.1 that there is some $q_{\delta} \in Y^{\omega}$ such that $d\left(q_{\delta}, y\right)=\varepsilon$ and such that for the starting directions $v_{\delta}$ of $y z_{\delta}$ resp. $w_{\delta}$ of $y q_{\delta}$ the inequality $d\left(v_{\delta}, w_{\delta}\right) \geq \frac{\pi}{2}$ holds.

Since the triangle $y z q$ is spherical we know $d\left(w, v_{\delta}\right)+d\left(v_{\delta}, v\right)=$ $d(v, w)=\frac{\pi}{2}$. Therefore from $\operatorname{rad}_{v}\left(S_{y}\right)=\frac{\pi}{2}$ and the fact that $S_{y} Y^{\omega}$ is one-dimensional we deduce that $d\left(w, w_{\delta}\right) \rightarrow \pi$. Now using a diagonal argument in $Y$ (or going to another ultraproduct $\left(Y^{\omega}\right)^{\omega}$ ) we obtain a point $q_{0}$ such that $y$ is an inner point of the geodesic $q q_{0}$.

This finishes the proof of Theorem 1.1.

\section{REFERENCES}

[BH99] M. R. Bridson and A. Haefliger. Metric spaces of non-positive curvature. Springer, 1999.

[BL04] A. Balser and A. Lytchak. Building-like spaces. Preprint; Arxiv: math.MG/0410437, 2004.

[FNS04] K. Fujiwara, K. Nagano, and T. Shioya. Fixed points sets of parabolic isometries of CAT(0)-spaces. Preprint; Arxiv: math.DG/0408347, 2004.

$[\mathrm{KL}] \quad$ B. Kleiner and B. Leeb. Invariant convex sets in symmetric spaces of noncompact type. Preprint.

[KL97] B. Kleiner and B. Leeb. Rigidity of quasi-isometries for symmetric spaces and Euclidean buildings. Inst. Hautes Études Sci. Publ. Math., 86:115197, 1997.

[Kle99] B. Kleiner. The local structure of length spaces with curvature bounded above. Math. Z., 231(3):409-456, 1999.

[LS97] U. Lang and V. Schroeder. Jung's theorem for Alexandrov spaces of curvature bounded above. Ann. Global Anal. Geom., 15(3):263-275, 1997.

[Lyt04] A. Lytchak. Rigidity of spherical buildings and joins. Preprint, 2004. 